de Bruijn, M.J. 180

Dejonckere, P.H. 171

Fourcin, A. 126

Fraile, R. 146

Fredouille, C. 146

Godino-Llorente, J.I. 146

Horáček, J. 137

Kob, M. 125, 171

Kuik, D.J. 180

Langendijk, J.A. 180

Laukkanen, A.-M. 137

Leemans, C.R. 180
Murphy, P. 137

Neuman, K. 125

Osma-Ruiz, V. 146

Quené, H. 180

Sáenz-Lechón, N. 146

Šidlof, P. 137

Stylianou, Y. 153

Švec, J.G. 137

ten Bosch, L. 180

Vasilakis, M. 153

Verdonck-de Leeuw, I.M. 180

\title{
Subject Index Vol. 61, No. 3, 2009
}

Acoustic analysis 180

Automatic detection of laryngeal pathologies 146

Beat spectrum 153

Biomechanics of voice modeling 137

Connected speech 126

Continuous speech 153

Fundamental frequency 137

Gender differences in voice 137

Head and neck cancer 180

Jitter 153

Mel-frequency cepstral coefficients 146

Muscular tension imbalance 171
Phonation type 137

Radiotherapy 180

Short-term jitter 153

Spectral jitter 153

Speech quality 180

Surgical reconstruction 180

Sustained vowels 146,153

Vibrating vocal folds, 3-dimensional computer model 171

Vocal fold nodules 171

- nodules 137

Voice irregularity measurement 126 